\title{
Determinants of health- related quality of life (HRQoL) among deaf and hard of hearing adults in Greece: a cross-sectional study
}

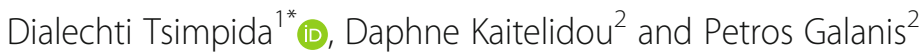

\begin{abstract}
Background: Hearing loss is an important public health issue, since it has a very negative impact on peoples' lives, irrespective of the age at which it develops. However, globally there is a noticeable lack of epidemiological data for health outcomes for people who are deaf and hard of hearing. In Greece, people with hearing disabilities are systematically not included in health policy and planning processes, despite there being a marked tendency for global efforts aimed at improving their quality of life.

Methods: The sample consisted of 140 adults with hearing loss ( $86 \mathrm{~d} /$ Deaf and 54 hard of hearing) and 97 normal hearing as the control group. We run data collection from April to June 2015, using the Greek version of the 36-Item Short Form Health Survey (SF-36v2). Socio-demographic and characteristics about non-medical determinants of health (tobacco and alcohol consumption levels, BMl and physical activity).were also collected and were analysed as possible determinants. Data analysis included bivariate and multivariate analyses such as linear regression models.

Results: Multivariate analyses identified that in all the SF-36v2 dimensions, the scores among deaf people were lower than those with normal hearing. Determinants included the hearing loss degree, educational level, body mass index, levels of physical activity, and alcohol consumption levels, while the variable "number of family members per household" was inversely associated with physical health summary scale score.

Conclusions: Improving knowledge of the health-related determinants that affect quality of life for the population with hearing loss is an important step in designing targeted services and interventions. In light of these findings, a special effort must be made to ensure the wellbeing of this population.
\end{abstract}

Keywords: Deaf, Hard of hearing, Deafness, Health-related quality of life, Determinants

\section{Background}

Hearing impairment, deafness or hearing loss is a partial or total inability to hear: it may be ranked as mild, moderate, moderately severe, severe or profound. Impairment may result from genetic causes, complications at birth, certain infectious diseases, chronic ear infections, the use of particular drugs, exposure to excessive noise

\footnotetext{
* Correspondence: dialechti.tsimpida@postgrad.manchester.ac.uk

${ }^{1}$ Manchester Centre for Audiology and Deafness (ManCAD), Division of Human Communication, Development and Hearing, School of Health Sciences, Faculty of Biology, Medicine and Health, University of Manchester, Manchester Academic Health Science Centre, A4.01 Ellen Wilkinson Building, Oxford Road, Manchester M13 9PL, UK

Full list of author information is available at the end of the article
}

and ageing [1]. Although the consequences of hearing loss are never obvious, hearing loss is a major global health challenge, as over $5 \%$ of the world's population 360 million people - has disabling hearing loss (hearing threshold of $41 \mathrm{~dB}$ or greater in the better ear) [1]. Hearing loss -irrespective of the age at which it develops has serious consequences for interpersonal communication, psychosocial well-being and individual quality of life $[2,3]$. In most countries, hearing loss is projected to be among the top ten burdens of disease by 2030 , with the associated detrimental social and economic effects, which will be greater in low-and middle-income countries [2].

(c) The Author(s). 2018 Open Access This article is distributed under the terms of the Creative Commons Attribution 4.0 International License (http://creativecommons.org/licenses/by/4.0/), which permits unrestricted use, distribution, and reproduction in any medium, provided you give appropriate credit to the original author(s) and the source, provide a link to the Creative Commons license, and indicate if changes were made. The Creative Commons Public Domain Dedication waiver (http://creativecommons.org/publicdomain/zero/1.0/) applies to the data made available in this article, unless otherwise stated. 
Despite the seriousness of this public health issue, there is a noticeable lack of epidemiological data for health outcomes for people who are deaf and hard of hearing [4]. However, the few studies that have focused specifically on this topic [5-11] showed that while the implications of hearing loss differ from person to person, it consistently has a negative impact on peoples' lives across numerous quality of life measures, including mental health, social functioning and general health.

In Greece, people with hearing disabilities are systematically excluded from health policy and planning processes, despite there being a marked tendency for global efforts aimed at improving their quality of life [4]. Furthermore, due to the severity of the economic downturn that Greece is facing, government grants for Greek Sign Language (GSL) interpretations have significantly reduced. That means that those who communicate via GSL have been forced to reduce their quality of life, due to communication barriers they face. From 2011 to date, the GSL users have the right to $25 \mathrm{~h}$ of free interpretation per year; whereas prior to 2011, the State fully paid interpretation costs for all their communication needs, and the available interpretation hours per person were unlimited. The contribution of GSL interpretation in the quality of life of Deaf/HH GSL users is enormous, since only via the presence of an interpreter do these people enjoy interactive and effective communication in all aspects of their life, covering all their communications needs, including use of and access to healthcare services (hospitals and clinics) [12]. In case GSL users consume their 25 free hours of interpretation, they then have to pay the interpretation costs making private payments, which are significantly higher when related to health issues. This is an important barrier for this population in accessing health services, with varying implications on their health-related quality of life (HRQoL).

Identifying determinants of the health-related quality of life of deaf and hard of hearing people is of great importance so as their health status can be improved. This study aims to fill a gap in the literature, by investigating the epidemiological profile of deaf and hard of hearing, so thatthe futurepolicy on their healthcare needs would be evidence-based. The objectives of this study included an assessment of the health-related quality of life of deaf and hard of hearing adults (aged 18-65), and the investigation of the factors that affect it.

\section{Methods}

\section{Participants}

A cross-sectional study was conducted, and the study population consisted of 140 young and middle-aged adults (18-65 years) with hearing loss ( $86 \mathrm{~d} /$ Deaf and 54 hard of hearing) and 97 hearing participants. There are several groups included within the broad "deaf and hard of hearing" category, as the population with hearing loss consists of subgroups with distinctly different cultural and communicational characteristics, which need to be separately examined. Factors that must be considered with Deaf/HH sub-populations include a) degree of hearing loss, b) age of onset of loss (pre-lingual/post-lingual), c) preferred language, and d) psychological issues [13]. Following a convention proposed by James Woodward [14], we use the lowercase deaf when referring to the audiological condition of not hearing, and the uppercase Deaf when referring to a particular group of deaf people who share a language - (GSL) - and a culture. The members of this group have adopted the GSL in their daily life, they use it as primarily for their communication and hold a set of beliefs about themselves and their connection to wider society. We distinguish them from, for example, those who find themselves losing their hearing because of illness, trauma or age; although those people share the condition of not hearing, they do not have access to the knowledge, beliefs and practices that make up the culture of Deaf people. Additionally, the distinction between the definition of "deaf" and "hard of hearing" is often given under different criteria, as it has strong ties with the individuals' ideological positions about education, social inclusion and/or rehabilitation of people with hearing loss [13].

In this study, the participants with hearing loss were categorised by combination of cultural self-identification, and their preferred method of communication, in order to self-identify themselves in one of the following five categories; a classification that fits best with the population with hearing loss in Greek culture.

1. Deaf - Persons referred to as Deaf (upper case D) typically belong to the Deaf Community and use GSL as a primary language [14].

2. deaf - Persons referred to as deaf (lower case d), that do not consider themselves members of the Deaf Community, although they may be severely or profoundly deaf, strive to identify themselves with hearing people and regard their hearing loss solely according to biomedical model of health, using non-signing communication [15].

3. Hard of hearing that communicate in terms of auditory-verbal approach (emphasis is given on strengthening the auditory channel through listening only - before any visual information is presented) [16].

4. Hard of hearing who communicate via lip-reading (a technique of understanding speech by visually interpreting the movements of the lips, faces and tongue) [17].

5. Hard of hearing who communicate using GSL as a preferred method of communication [14]. 
Regarding the sampling method, there is no accurate census of deaf and hard of hearing in Greece since members of the subcategories of this population are extremely difficult to be identified, except from the members of the first subcategory who are registered in deaf clubs- Thus, the probability or random sampling was not feasible and therefore a convenience sampling technique was used. In regards to the deaf and hard of hearing participants that communicate via GSL, the recruitment was done by visiting the 5 Deaf Clubs-members of the Hellenic Federation of the Deaf which are located in Attica (4 in Athens and one in Korydallos). In addition, the Hellenic Federation of the Deaf embedded a link of an online survey in their website. This helped the wide distribution of the questionnaire and to reach the selected population, as deaf and hard of hearing individuals in Greece use this website to get local news and information, even if they do not communicate via sign-language. We also had placed a link on social networks, to provide also quick access to participants with similar demographics that had normal hearing, according to their answers in self-reported hearing difficulties.

\section{Materials}

The questionnaire consisted of three sections. The first section explained the purpose of the questionnaire and provided a contact name and address for any enquiries regarding the study. The second and third section consisted of the 36-item Short Form Health Survey (SF-36v2), and questions on standard socio-demographic data such as gender, age, marital status, number of family members per household, complete educational status, work status, income and non-medical determinants of health (tobacco and alcohol consumption, BMI and physical activity). The SF-36 Health Survey Version 2, for the measurement of the perceived health-related quality of life, is a widely used questionnaire. The SF-36v2 Health Survey health domain scales are: Physical Functioning, Role-Physical, Bodily Pain, General Health, Vitality, Social Functioning, Role-Emotional, Mental Health. Higher scores across each subscale indicate greater HRQoL. The psychometrically-based physical component summary and mental component summary scores are also provided. The SF-36 has been translated and its psychometric properties tested for a Greek population by Pappa et al. [18].

A pilot study with 6 deaf adults was carried out, in order to check the comprehensibility of the questionnaire, as for the Sign Language users the Greek language is being perceived as a second language, and it is being taught to the Schools of the Deaf along with the GSL, which is their first language. In addition, they are obligated to learn only the written form of the Greek language and not the oral form according to the Greek Law
$\mathrm{N}^{\mathrm{o}} 3699 / 2008$. The 6 pilot subjects were representative of the overall Deaf signers that were enrolled into this project, as they represent them unanimously on a regular basis in the Community, and they have a deep understanding of their educational and other needs. Face validity of the questionnaire was very good and no significant corrections were made.

\section{Procedure}

The data collection was done in person, so that the questions were presented in GSL from the first author (TD) when it was necessary, in order to improve accessibility and minimise the language barrier for Deaf, as they completed a lengthy written questionnaire in a second language. The completion of the questionnaire by the deaf and hard of hearing non-users of GSL and by the hearing participants was done in person, following a convenience sampling technique.

All participants were informed about the aim and procedures of this study (additionally in GSL by TD if it was necessary) and gave their consent. Personal data of the participants were not registered at any stage of the study. Participation in this study was voluntary and anonymous. The previous engagement of the first author in the Deaf community, as she has a National Certification in GSL and participates in their community activities, reinforced the feelings of trust and comfort of the participants, leading to a high response rate $(91 \%)$. The response rate for the hearing participants was 93\%.

\section{Statistical analysis}

Categorical variables are presented as absolute (n) and relative (\%) frequencies, while quantitative variables are presented as mean (standard deviation, SD) or median (range). The Kolmogorov-Smirnov test and normal plots were used to test the normality of quantitative variable distribution.

Firstly, we performed bivariate analyses between sociodemographic and non-medical characteristics and scores on SF-32v2 scales. Bivariate associations between categorical variables were assessed with chi-square test, while between categorical and ordinal variables with chi-square trend test. Student's t-test and analysis of variance were used for the association between categorical variables and a continuous one that followed normal distribution. Also, we used Kruskal-Wallis test to assess the relation between categorical variables and a continuous one that did not follow normal distribution. We used Pearson's and Spearman's correlation coefficient to find out the correlation between continuous variables that followed and did not follow normal distribution respectively.

Scores on SF-32v2 scales were the dependent variables. In case that $>2$ independent variables were associated 
with scores on SF-32v2 scales $(p<0.2)$ in bivariate analyses, then multivariate linear regression analysis was applied, using the backward stepwise linear regression model in order to eliminate confounding. For multivariate linear regression models, coefficients' beta values, 95\% confidence intervals and $p$ values are presented.

'Work status' was not used as an independent variable due to the small number of participants in several categories $(n \leq 10)$.

The five designed subcategories of population with hearing loss were not finally used as independent variables due to the exceptionally small number of participants in several categories $(n \leq 10)$. Therefore, the classification was limited to the three bigger categories (deaf/ hard of hearing/hearing) and it was not possible to consider the exact language preferences of the participants, but only their cultural self-identification. That means that the "deaf category" in the statistical analysis finally included those that self-identified themselves as severely or profoundly deaf and used to communicate via GSL or non-signing communication (category No1 and No2), while the "hard of hearing category" included those that self-identified themselves as having mild, moderate or moderately severe hearing loss and used to communicate in terms of auditory-verbal approach or via lip-reading (category No3 and No4).

The two-tailed significance level was set $\leq 0.05$. Data were analyzed using IBM SPSS 21.0 (IBM Corp. Released 2012. IBM SPSS Statistics for Windows, Version 21.0. Armonk, NY: IBM Corp).

\section{Results}

\section{Socio-demographic characteristics}

Socio-demographic characteristics are presented in Table 1. The two-tailed significance level was set $\leq 0.05$. For the $91.9 \%$ of deaf $(n=79)$ the GSL was the preferred method of communication, while for the $8.1 \%(n=7)$ was not. Among the 54 hard of hearing, the $20.4 \%$ ( $n=$ 11) preferred to communicate via the oral method, the $50 \%(n=27)$ via the lip-reading technique and the $29.6 \%$ $(n=16)$ via the GSL.

\section{Non-medical determinants of health}

Non-medical determinants of health are shown in Table 2. The mean of BMI was higher for deaf compared to hard of hearing and hearing people $(p=0.02)$. There was a higher proportion of overweight $(55.8 \%)$ and obese people (20.9\%) among deaf and hard of hearing adults $(55.6 \%$ and $18.5 \%$ accordingly), while almost half of the hearing participants $(44.3 \%)$ had a normal BMI $(p=0.004)$ (Table 2).

Concerning tobacco consumption, the median number of cigarettes smoked per day was higher for hard of hearing and deaf compared to the hearing participants $(p<0.001)$. Besides, the median number of years of smoking was higher for hard of hearing and deaf compared to the hearing participants $(p=0.01)$.

The median hours of physical activity per week was higher for hearing and hard of hearing compared to deaf participants $(p<0.001)$. The median number of units of alcohol per week was higher for deaf compared to hard of hearing and hearing participants $(\mathrm{p}<0.001)$.

\section{Short-form health survey}

Cronbach's coefficient alpha across the domains of SF-36v2 ranging from 0.91 to 0.93 , indicating high internal consistency reliability.

The bivariate associations between all independent variables and scores on SF-32v2 scales are presented in Table 3 . In case that $>2$ independent variables were associated with scores on SF-32v2 scales $(p<0.2)$ in bivariate analyses, then multivariate linear regression analysis was applied, using the backward stepwise linear regression model in order to eliminate confounding.

Multivariate linear regression analyses revealed a statistically significant relation between hearing loss and lower SF-36v2 scores across subscales, even after controlling for confounders. If the confidence interval did not include the value of zero effect, it was assumed that there was a statistically significant result (Table 4). Concerning the physical health domain, deaf participants had a lower Physical Functioning score compared to hearing, a lower Role Physical Score compared to hearing and hard of hearing, a lower Bodily Pain score compared to hearing, and a lower Physical Health Component Summary score compared to hearing. Concerning the physical health domain, deaf participants had a lower Vitality score compared to hearing and hard of hearing, a lower Social Functioning score compared to hearing and hard of hearing, a lower Role Emotional score compared to hearing and hard of hearing, a lower Mental Health score compared to hearing and hard of hearing, and a lower Mental Health Component Summary score compared to hearing and hard of hearing.

Respondents with a higher degree of hearing loss had lower scores in all SF-36 sections, excluding the General Health score, while decreased body mass index (BMI) was associated with increased scores in all SF-36 sections, excluding the Bodily Pain score.

Higher educational level was associated with increased scores in Physical Functioning, Role Physical, Bodily Pain, General Health, Social Functioning and Physical Health Component Summary scores and increased physical activity was associated with increased scores in Physical Functioning, General Health, Role Emotional and Physical Health Component Summary scores.

Finally, decreased alcohol consumption was associated with increased scores in all the SF-36v2 dimensions except social functioning and decreased number of family 
Table 1 Participants socio-demographic characteristics, N=237

\begin{tabular}{|c|c|c|c|c|}
\hline Variable & Hearing & Hard of Hearing & Deaf & $P$-value \\
\hline Gender & & & & $0.02^{* a}$ \\
\hline Women & $71(73.2)$ & $32(59.3)$ & $46(53.5)$ & \\
\hline Men & $26(26.8)$ & $22(40.7)$ & $40(46.5)$ & \\
\hline Age (years) ${ }^{b}$ & $36.3(9.4)$ & $41.4(11.8)$ & $38.1(10.7)$ & $0.02^{* c}$ \\
\hline Marital Status & & & & $0.006^{* * a}$ \\
\hline Unmarried & $42(43.3)$ & $16(29.6)$ & $37(43.0)$ & \\
\hline In cohabitation & $13(13.4)$ & $14(25.9)$ & $11(12.8)$ & \\
\hline Married & $38(39.2)$ & $14(25.9)$ & $20(23.3)$ & \\
\hline Divorced & $3(3.1)$ & $7(13.0)$ & $13(15.1)$ & \\
\hline Widowed & $1(1.0)$ & $3(5.6)$ & $5(5.8)$ & \\
\hline Number of family members per household ${ }^{e}$ & $3.5(1.3)$ & $2.7(1.2)$ & $2.6(1.2)$ & $<0.001^{* * * c}$ \\
\hline Existence of hearing person in household ${ }^{a}$ & & $38(70.4)$ & $37(43)$ & $0.002^{* * a}$ \\
\hline Educational attainment & & & & $<0.001^{* * * d}$ \\
\hline Junior High & $0(0.0)$ & $4(7.4)$ & $11(12.8)$ & \\
\hline High School & $23(23.7)$ & $19(35.2)$ & $59(68.6)$ & \\
\hline College & $9(9.3)$ & $3(5.6)$ & $2(2.3)$ & \\
\hline Technological Educational Institution (TEI) & $13(13.4)$ & $5(9.3)$ & $0(0)$ & \\
\hline University & $31(32.0)$ & $11(20.4)$ & $10(11.6)$ & \\
\hline Master's/Doctorate degree & $21(21.6)$ & $12(22.2)$ & $4(4.7)$ & \\
\hline Work Status & & & & $0.001^{* * a}$ \\
\hline Unemployed & $10(10.3)$ & $8(14.8)$ & $21(24.4)$ & \\
\hline Household keeper & $2(2.1)$ & $3(5.6)$ & $5(5.8)$ & \\
\hline Income collection & $2(2.1)$ & $0(0)$ & $1(1.2)$ & \\
\hline Student & $10(10.3)$ & $1(1.9)$ & $5(5.8)$ & \\
\hline Unskilled worker & $0(0)$ & $4(7.4)$ & $6(7.0)$ & \\
\hline Private sector employee & $31(32.0)$ & $10(18.5)$ & $30(34.9)$ & \\
\hline Public sector employee & $27(27.8)$ & $18(33.3)$ & $16(18.6)$ & \\
\hline Entrepreneur & $12(12.4)$ & $6(11.1)$ & $0(0)$ & \\
\hline Retired & $3(3.1)$ & $4(7.4)$ & $2(2.3)$ & \\
\hline Family annual income (euro) ${ }^{e}$ & $20,000(195,000)$ & $15,000(75,000)$ & $15,000(49,000)$ & $<0.001^{* * *^{f}}$ \\
\hline
\end{tabular}

Values are expressed as $n$ (\%) unless otherwise is indicated

${ }^{*} p$ value 0.01 to 0.05 (significant)

**p value 0.001 to 0.01 (very significant)

${ }^{* * *} \mathrm{p}$ value 0.0001 to 0.001 (extremely significant)

${ }^{a} x^{2}$ test

${ }^{\mathrm{b}}$ Mean (standard deviation)

${ }^{\mathrm{c}}$ Analysis of variance

${ }^{d} x^{2}$ trend test

eMedian (range)

${ }^{f}$ Kruskal-Wallis test

members per household was associated with increased Physical Health Component Summary score.

\section{Discussion}

Previous studies have revealed that disabling hearing loss, which refers to hearing loss greater than $41 \mathrm{~dB}$ in the better hearing ear in adults, is associated with poorer physical health [19-23] and lower levels of mental health
[24-34]. This study adds to the limited number of studies which associated the degree of hearing loss with poorer HRQoL in young and middle-aged adults [5, 7 , 9-11, 32], while according to our knowledge it is the only one where the HRQoL was evaluated via the 36-Item Short Form Health Survey (SF-36) in this specific population, and did not concern older adults [35, 36]. The findings of our study showed that respondents 
Table 2 Participants' non-medical determinants of health, $N=237$

\begin{tabular}{|c|c|c|c|c|}
\hline Variable & Hearing & Hard of Hearing & Deaf & P-value \\
\hline Tobacco consumer & & & & $0.13^{\mathrm{a}}$ \\
\hline Current & $38(39.2)$ & $20(37.0)$ & $48(55.8)$ & \\
\hline Former & 18 (18.6) & $9(16.7)$ & $10(11.6)$ & \\
\hline Never & $41(42.3)$ & $9(46.3)$ & $28(32.6)$ & \\
\hline Number of cigarettes smoked per day ${ }^{e}$ & $11(59)$ & $20(58)$ & $20(35)$ & $<0.001^{* * *^{f}}$ \\
\hline Years of smoking ${ }^{e}$ & $12(37)$ & $20(43)$ & $18(39)$ & $0.01^{* f}$ \\
\hline Alcohol consumption (units of alcohol per week) ${ }^{e}$ & $1(10)$ & $2(10)$ & $3.5(15)$ & $<0.001^{* * * f}$ \\
\hline Body Mass Index (BMI) ${ }^{b}$ & $26.1(4.6)$ & $27.4(4.2)$ & $27.9(4.1)$ & $0.02^{* c}$ \\
\hline BMI Classification & & & & $0.004^{* * d}$ \\
\hline Underweight & $2(2.1)$ & $0(0)$ & $0(0)$ & \\
\hline Normal & $43(44.3)$ & $14(25.9)$ & $20(23.3)$ & \\
\hline Overweight & $37(38.1)$ & $30(55.6)$ & $48(55.8)$ & \\
\hline Obese & $15(15.5)$ & $10(18.5)$ & $18(20.9)$ & \\
\hline Physical activity (hours per week) ${ }^{e}$ & $2(10)$ & $2(10)$ & $0(8)$ & $<0.001^{* * * f}$ \\
\hline 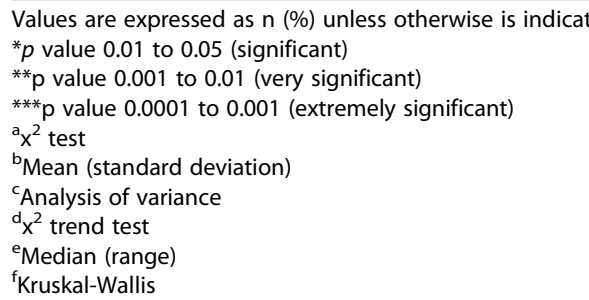 & & & & \\
\hline
\end{tabular}

with higher degrees of hearing loss had lower scores in physical functioning, role physical, bodily pain, vitality, social functioning, role emotional, mental health domains, Physical Health and Mental Health Component.

Decreased numbers of household individuals, and particularly individuals living alone, has been associated with lower mental health levels, which is associated with depression among adults with hearing loss $[31,34]$. However, in our study, decreased numbers of household individuals was associated with increased Physical Health Component Summary score. One possible explanation for this paradox is the better ability of participants to pay privately for health and interpreting services if a smaller number of persons cohabiting shares a low household's income. This may happen as the very high percentage of private payments is a significant characteristic of the mixed financial resources of the Greek health care system [37].

The findings of our study showed that increased education was associated with increased scores in Physical Functioning, Role Physical, Bodily Pain, General Health, Social Functioning and Physical Health Component Summary and are in compliance with previous studies [10, 19, 21, 36, 38]. McKee et al. [38], have also supported that educational attainment is more important than higher income as a physical health protecting factor.
Concerning the variable tobacco consumption, in this study the $55.8 \%$ of deaf were current tobacco consumers, while in the Health Interview Survey [39] the rate for the general population was $32.5 \%$. The median number of cigarettes smoked per day was higher in hard of hearing and deaf compared to hearing adults, but the multivariate linear regression did not reveal a statistically significant correlation between the tobacco consumption and health-related quality of life. Conversely, Schoenborn \& Heyman [20], had revealed that adults with lower or higher level of hearing loss used to smoke at higher rates than the hearing population (40\% instead of $24 \%$ ), while other studies [19, 22, 23] have shown lower rates of tobacco consumption among deaf or hard of hearing compared to hearing individuals. In a previous study [34], tobacco consumption (current/former tobacco consumer) has been associated with increased risk to mental health problems, while is associated with depression among adults with hearing loss.

Also, we found that decreased alcohol consumption was associated with increased scores in all the SF-36v2 dimensions except social functioning. The higher number of alcohol units per week has been associated also in a previous study [34] with lower mental health levels, which is associated with depression among adults with hearing loss [31, 34]. 


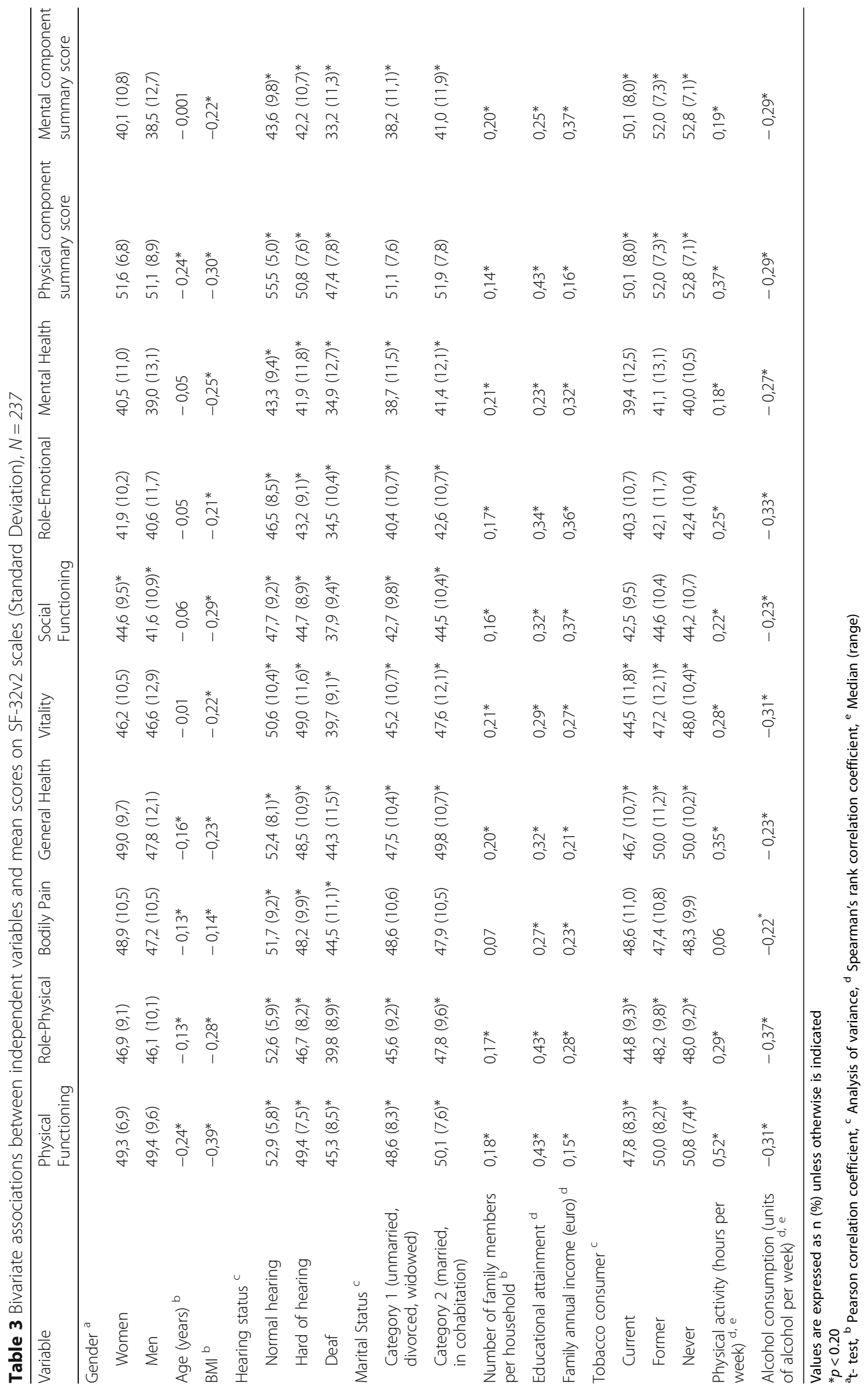


Table 4 Multivariate linear regression analysis with SF-36v2 domains' scores as dependent variables

\begin{tabular}{|c|c|c|}
\hline & b coefficient $(95 \% \mathrm{Cl})$ & $P$-value \\
\hline \multicolumn{3}{|l|}{ Physical Functioning } \\
\hline BMI & $-0.58(-0.76$ to -0.40$)$ & $<0.001$ \\
\hline Hearing compared to deaf & 2.06 (0.29 to 3.83 ) & 0.023 \\
\hline Educational level & 1.05 (0.53 to 1.58$)$ & $<0.001$ \\
\hline Physical Activity (hours per week) & 1.09 (0.73 to 1.44$)$ & $<0.001$ \\
\hline Alcohol consumption (units of alcohol per week) & $-0.37(-0.69$ to -0.05$)$ & 0.023 \\
\hline \multicolumn{3}{|l|}{ Role Physical } \\
\hline BMI & $-0.40(-0.62$ to -0.19$)$ & $<0.001$ \\
\hline Hearing compared to deaf & 9.52 (6.95 to 12.09$)$ & $<0.001$ \\
\hline Hard of hearing compared to deaf & 4.51 (1.86 to 7.17$)$ & 0.001 \\
\hline Educational level & $0.88(0.23$ to 1.52$)$ & 0.008 \\
\hline Alcohol consumption (units of alcohol per week) & $-0.45(-0.84$ to -0.05$)$ & 0.027 \\
\hline \multicolumn{3}{|l|}{ Bodily Pain } \\
\hline Hearing compared to deaf & 3.18 (0.31 to 6.05$)$ & 0.03 \\
\hline Alcohol consumption (units of alcohol per week) & $-0.78(-1.30$ to -0.26$)$ & 0.004 \\
\hline \multicolumn{3}{|l|}{ General Health } \\
\hline BMI & $-0.44(-0.71$ to -0.16$)$ & 0.002 \\
\hline Educational level & 0.91 (0.11 to 1.70$)$ & 0.024 \\
\hline Physical Activity (hours per week) & $-0.96(-1.44$ to -0.48$)$ & $<0.001$ \\
\hline Alcohol consumption (units of alcohol per week) & $1.05(0.49$ to 1.61$)$ & $<0.001$ \\
\hline \multicolumn{3}{|l|}{ Vitality } \\
\hline BMI & $-0.40(-0.70$ to -0.10$)$ & 0.009 \\
\hline Hearing compared to deaf & 7.86 (4.48 to 11.22$)$ & $<0.001$ \\
\hline Hard of hearing compared to deaf & 7.45 (3.86 to 11.09$)$ & $<0.001$ \\
\hline Alcohol consumption (units of alcohol per week) & $-0.55(-1.08$ to -0.03$)$ & 0.039 \\
\hline \multicolumn{3}{|l|}{ Social Functioning } \\
\hline BMI & $-0.52(-0.78$ to -0.26$)$ & $<0.001$ \\
\hline Hearing compared to deaf & 7.45 (4.50 to 10.40$)$ & $<0.001$ \\
\hline Hard of hearing compared to deaf & 5.54 (2.37 to 8.72$)$ & 0.001 \\
\hline Educational level & $0.78(0.03$ to 1.54$)$ & 0.043 \\
\hline \multicolumn{3}{|l|}{ Role Emotional } \\
\hline $\mathrm{BMl}$ & $-0.32(-0.58$ to -0.05$)$ & 0.022 \\
\hline Hearing compared to deaf & 9.04 (5.99 to 12.10$)$ & $<0.001$ \\
\hline Hard of hearing compared to deaf & $6.80(3.52$ to 10.07$)$ & $<0.001$ \\
\hline Alcohol consumption (units of alcohol per week) & $-0.60(-1.08$ to -0.13$)$ & 0.013 \\
\hline Physical Activity (hours per week) & 0.55 (0.01 to 1.09$)$ & 0.046 \\
\hline \multicolumn{3}{|l|}{ Mental Health } \\
\hline BMI & $-0.54(-0.86$ to -0.22$)$ & 0.001 \\
\hline Hearing compared to deaf & 4.79 (1.24 to 8.34$)$ & 0.008 \\
\hline Hard of hearing compared to deaf & $5.02(1.20$ to 8.84$)$ & 0.010 \\
\hline Alcohol consumption (units of alcohol per week) & $-0.97(-1.53$ to -0.41$)$ & 0.001 \\
\hline
\end{tabular}


Table 4 Multivariate linear regression analysis with SF-36v2 domains' scores as dependent variables (Continued)

\begin{tabular}{|c|c|c|}
\hline & b coefficient $(95 \% \mathrm{Cl})$ & Test ( $p$-value) \\
\hline \multicolumn{3}{|l|}{ Physical Health Component Summary Score } \\
\hline BMI & $-0.39(-0.57$ to -0.21$)$ & $<0.001$ \\
\hline Hearing compared to deaf & 3.87 (2.05 to 5.68$)$ & $<0.001$ \\
\hline Educational level & 1.07 (0.54 to 1.59$)$ & $<0.001$ \\
\hline Alcohol consumption (units of alcohol per week) & $-0.51(-0.83$ to 0.18$)$ & 0.002 \\
\hline Physical Activity (hours per week) & 0.64 (0.28 to 0.99$)$ & 0.001 \\
\hline Number of family members per household & $-0.74(-1.39$ to -0.09$)$ & 0.026 \\
\hline \multicolumn{3}{|l|}{ Mental Health Component Summary Score } \\
\hline BMI & $-0.43(-0.73$ to 0.12$)$ & 0.006 \\
\hline Hearing compared to deaf & $7.62(4.21$ to 11.02$)$ & $<0.001$ \\
\hline Hard of hearing compared to deaf & 7.52 (3.86 to 11.17$)$ & $<0.001$ \\
\hline Alcohol consumption (units of alcohol per week) & $-0.70(-1.24$ to -0.17$)$ & 0.010 \\
\hline
\end{tabular}

Respectively, we found that $55.8 \%$ and $20.9 \%$ of deaf were overweight and obese, while the percentages in hearing people were $38.1 \%$ and $15.5 \%$. In the Health Interview Survey [39], percentages in the general population were $39.4 \%$ and $16.9 \%$. In addition, we found that decreased body mass index was associated with increased scores on all SF-32v2 scales, except for the bodily pain score. Weight that is higher than what is considered as a healthy weight, has been associated also in previous studies with lower level of health-related quality of life, in both physical and mental health domains for population with hearing loss $[19,20,22,23,34]$.

Also, in our study, increased physical activity was associated with increased scores in physical functioning, general health, role emotional and physical health component summary. In previous studies the lower physical activity levels have also been associated with population with hearing loss [19, 20].

\section{Limitations}

There are several limitations when conducting a study among deaf and hard of hearing participants. However, the previous engagement of the first author (TD) with the Deaf community, allowed many of these methodological issues being eliminated as much as possible.

The main limitation of our study is that we did not use an additional specific questionnaire for hearing loss - for example the Hearing Handicap Inventory for Adults (HHIA) - to identify problems that hearing loss may cause to participants, as there have been expressed doubts in literature concerning differential performance of SF-36 items in healthy adults with and without functional limitations [40]. However, this multidimensional 36-item Health Survey has been used widely across a range of diseases and treatment groups. Even if certain questions may not intuitively relate to hearing impairment, it has been argued that allows the direct comparison of the effects of several determinants including hearing impairment on the health-related quality of life of populations [35]. Moreover, we considered the SF-36v2 sufficient as it has been translated and its psychometric properties have been tested for a Greek population [18], while the Hearing Handicap Inventory for Adults (HHIA) has not. Another limitation was that the deaf population consists of subgroups with different cultural and communicational characteristics, which need to be separately examined. In this study, the five designed subcategories of population with hearing loss were not used as independent variables. The reason was the exceptionally small number of participants in several categories $(n \leq 10)$. As a result, the classification was limited to the three bigger categories (deaf/ hard of hearing/hearing) and it was not possible to take into consideration the language preferences of the participants, but only their cultural self-identification. This should be considered by future studies aiming to assess the health-related quality of life in a larger sample of population with hearing loss, as e.g. the Deaf community struggles with a number of socioeconomic-based health disparities, many of which are not directly associated with the degree of hearing loss.

Validating and standardizing a conceptual model [11] of health-related quality of life among people with a range of functional hearing and language preferences, including GSL, that delineates the relationships between health status (self-acceptance, coping with limitations), intrinsic (functional communication skills, navigating barriers/self-advocacy, resilience) and extrinsic (acceptance by others, access to information, educating others) factors in their influence on quality of life outcomes among young and middle-aged adults with hearing loss, should be considered for the future. 


\section{Conclusions}

As hearing loss is an important global health concern, health-related quality of life among deaf and hard of hearing adults must be estimated as a factor of great importance. In Greece, people with hearing disabilities are systematically excluded in health policy and planning processes, despite there is a marked tendency for global efforts aimed at improving their quality of life.

Identifying determinants of the health- related quality of life of deaf and hard of hearing people is decisive, so as their health condition can be improved. The findings of this study suggest that hearing loss is capable of contributing to HRQoL deficits in Greek deaf and hard of hearing adults, as their health is poorer than that of the general population, with probable underdiagnosis and undertreatment of chronic conditions, putting them at risk of preventable ill health.

Health information is not available in sign language for Deaf people and it may affect their skills in taking decisions in everyday life concerning health promotion. That is being reflected in several determinants (such as tobacco and alcohol consumption, BMI and physical activity). Programs that raise health knowledge in Deaf communities could contribute to improve their skills in take decisions in everyday life concerning the promotion of a healthy lifestyle. This could help in tackling health inequalities for people with hearing loss and in improving their quality of life.

Future research should involve a more extensive population with hearing loss, in order to produce health statistics of deaf and hard of hearing populations, so as they can be included in the priorities for improving health and chronic disease prevention programs and stop therefore this health condition from being a "silent epidemic" [4].

\section{Abbreviations}

GSL: Greek sign language

\section{Acknowledgements}

This study was part of the first author's master's thesis, completed at the Open University of Cyprus.

\section{Author details}

DT: Manchester Centre for Audiology and Deafness (ManCAD), Division of Human Communication, Development \& Hearing, School of Health Sciences, Faculty of Biological, Medical and Health Sciences, University of Manchester, UK.

\section{Funding}

This research received no specific grant from any funding agency in the public, commercial, or not-for-profit sectors.

\section{Availability of data and materials}

The datasets used and/or analysed during the current study are available from the corresponding author on reasonable request.

\section{Authors' contributions}

DT made substantial contributions to the conception, design, acquisition of data, analysis and interpretation of data, and has been involved in drafting the manuscript. DK has made substantial contributions to the conception and design, and has been involved in critically revising the manuscript. PG has made substantial contributions to the analysis and interpretation of the data, and has been involved in critically revising the manuscript. All authors have given final approval of the version to be published, and have agreed to be accountable for all aspects of the work in ensuring that questions related to the accuracy or integrity of any part of the work are appropriately investigated and resolved.

\section{Ethics approval and consent to participate}

All procedures performed in this study involving human participants were in accordance with the ethical standards of the institutional and/or national research committee and with the 1964 Helsinki declaration and its later amendments or comparable ethical standards. The study protocol and the questionnaire were approved by the Hellenic Federation of the Deaf, which is the official representative association of deaf and hard of hearing people in Greece, with the reference number 435/13-3-2015. Also, written license for the use of SF-36 was taken (Lic. No.: SLA QM027680 - CT164283/ OP040288). Questionnaires were distributed and collected from April 2015 to June 2015. All participants were informed about the aim and procedures of this study (additionally in GSL if it was necessary) and gave their consent.

Consent for publication

Not applicable.

\section{Competing interests}

The authors declare that they have no competing interests.

\section{Publisher's Note}

Springer Nature remains neutral with regard to jurisdictional claims in published maps and institutional affiliations.

\section{Author details}

${ }^{1}$ Manchester Centre for Audiology and Deafness (ManCAD), Division of Human Communication, Development and Hearing, School of Health Sciences, Faculty of Biology, Medicine and Health, University of Manchester, Manchester Academic Health Science Centre, A4.01 Ellen Wilkinson Building, Oxford Road, Manchester M13 9PL, UK. ${ }^{2}$ Center for Health Services Management and Evaluation, Department of Nursing, National \& Kapodistrian University of Athens, Athens, Greece.

Received: 19 March 2018 Accepted: 7 August 2018

Published online: 08 October 2018

\section{References}

1. World Health Organization. Deafness and hearing loss, Fact sheet $N^{\circ} 300$. 2015. Assessed 7 Mar 2018.

2. Mathers C. The global burden of disease: 2004 update. Geneva: WHO; 2008. http://www.who.int/healthinfo/global_burden_disease/GBD_report 2004update_full.pdf, Accessed 7 Mar 2018

3. World Health Organization. Millions of people in the world have hearing loss that can be treated or prevented. Geneva: World Health Organization; 2013. p. 1-17. http://www.who.int/pbd/deafness/news/ Millionslivewithhearingloss.pdf, Accessed 7 Mar 2018

4. Hearing loss: an important global health concern. Lancet. 2016;387(10036): 2351. https://doi.org/10.1016/S0140-6736(16)30777-2.

5. Ringdahl A, Grimby A. Severe-profound hearing impairment and healthrelated quality of life among post-lingual deafened Swedish adults. Scand Audiol. 2000;29(4):266-75.

6. Fellinger J, Holzinger D, Dobner U, Gerich J, Lehner R, Lenz G, Goldberg D. An innovative and reliable way of measuring health-related quality of life and mental distress in the deaf community. Soc Psychiatry Psychiatr Epidemiol. 2005;40(3):245-50.

7. Fellinger J, Holzinger D, Gerich J, Goldberg D. Mental distress and quality of life in the hard of hearing. Acta Psychiatr Scand. 2007;115(3):243-5.

8. Monzani D, Galeazzi GM, Genovese E, Marrara A, Martini A. Psychological profile and social behaviour of working adults with mild or moderate hearing loss. Acta Otorhinolaryngol Ital. 2008;28(2):61-6.

9. Hallberg LR, Hallberg U, Kramer SE. Self-reported hearing difficulties, communication strategies and psychological general well-being (quality of 
life) in patients with acquired hearing impairment. Disabil Rehabil. 2008; 30(3):203-12.

10. Henning MA, Krägeloh CU, Sameshima S, Shepherd D, Shepherd G, Billington R. Access to New Zealand sign language interpreters and quality of life for the deaf: a pilot study. Disabil Rehabil. 2011;33(25-26):2559-66.

11. Kushalnagar P, McKee M, Smith SR, Hopper M, Kavin D, Atcherson SR. Conceptual model for quality of life among adults with congenital or early deafness. Disabil Health J. 2014;7(3):350-5.

12. Steinberg AG, Barnett S, Meador HE, Wiggins EA, Zazove P. Health care system accessibility. Experiences and perceptions of deaf people. J Gen Intern Med. 2006;21(3):260-6.

13. Meador HE, Zazove P. Health care interactions with deaf culture. J Am Board Fam Pract. 2005;18(3):218-22.

14. Lampropoulou V. (Ed.) Society and the deaf: community and deaf culture. 1st module of training. Program EPEAEK, Patras: Department of Elementary Education. University of Patras Publications. 1999.

15. Crystal D. The Cambridge encyclopedia of language. 3rd ed. Cambridge: University Press; 2010.

16. Cole EB, Flexer $\mathrm{C}$. The two main Listening and Spoken Language approaches, historically, have been the Auditory-Verbal Approach (AV) and the Auditory-Oral Approach (A-O). In: Children with hearing loss: Developing listening and talking, birth to six: Plural Publishing; 2015. ISBN 978-1-59756-379-6

17. Woodhouse L, Hickson L, Dodd B. Review of visual speech perception by hearing and hearing-impaired people: clinical implications. Int J Lang Commun Disord. 2010;44(3):253-70. https://doi.org/10.1080/ 13682820802090281.

18. Pappas E, Kontodimopoulos N, Niakas D. Validating and norming of the Greek SF-36 health survey. Qual Life Res. 2005;14:1433-8.

19. Woodcock K, Pole JD. Health profile of deaf Canadians: analysis of the Canada community health survey. Can Fam Physician. 2007 Dec;53(12):2140-1.

20. Schoenborn CA, Heyman K. Health disparities among adults with hearing loss: United States, 2000-2006, NCfHS NCHS, NCHS Health EStat, vol. 2015. Atlanta: Centers for Disease Control and Prevention (CDC); 2008. p. 1-14.

21. Alexander A, Ladd P, Powell S. Deafness might damage your health. Lancet. 2012;379(9820):979-81.

22. SignHealth. A Report into the Health of Deaf People (detailed version of Sick Of It, for professionals). 2014. http://www.signhealth.org.uk/sick-of-itreport-professionals/ Accessed 7 Mar 2018.

23. Emond A, Ridd M, Sutherland H, Allsop L, Alexander A, Kyle J. The current health of the signing deaf community in the UK compared with the general population: a cross-sectional study. BMJ Open. 2015;5(1):e006668.

24. Zazove P, Niemann LC, Gorenflo DW, Carmack C, Mehr D, Coyne JC, Antonucci T. The health status and health care utilization of deaf and hardof-hearing persons. Arch Fam Med. 1993;2(7):745-52.

25. Bridgman G, Macpherson B, Rako M, Campbell J, Manning V, Norman-Kelly T. A national epidemiological survey of mental illness in the New Zealand Deaf community. In: Hjortsö T, von der Lieth L, Carlsen C, editors. Mental health services for deaf people: a worldwide perspective. Devon: European Society for Mental Health and Deafness; 2000. p. 216-34.

26. de Graaf R, Bijl RV. Determinants of mental distress in adults with a severe auditory impairment: differences between prelingual and postlingual deafness. Psychosom Med. 2002;64:61-70.

27. Fellinger J, Holzinger D, Dobner U, Gerich J, Lehner R, Lenz G, Goldberg D. Mental distress and quality of life in a deaf population. Soc Psychiatry Psychiatr Epidemiol. 2005;40(9):737-42.

28. Helvik AS, Jacobsen G, Hallberg LR. Psychological well-being of adults with acquired hearing impairment. Disabil Rehabil. 2006;28(9):535-45.

29. Kvam MH, Loeb M, Tambs K. Mental health in deaf adults: symptoms of anxiety and depression among hearing and deaf individuals. J Deaf Stud Deaf Educ. 2007;12(1):1-7.

30. Sheppard K, Badger T. The lived experience of depression among culturally deaf adults. J Psychiatr Ment Health Nurs. 2010;17(9):783-9.

31. Gopinath B, Hickson L, Schneider J, McMahon CM, Burlutsky G, Leeder SR, Mitchell P. Hearing-impaired adults are at increased risk of experiencing emotional distress and social engagement restrictions five years later. Age Ageing. 2012;41(5):618-23.

32. Fellinger J, Holzinger D, Schoberberger R, Lenz G. Psychosocial characteristics of deaf people: evaluation of data from a special outpatient clinic for the deaf. Nervenarzt. 2005:76(1):43-51. German
33. Fellinger J, Holzinger D, Pollard R. Mental health of deaf people. Lancet. 2012:379(9820):1037-44.

34. Li CM, Zhang X, Hoffman HJ, Cotch MF, Themann CL, Wilson MR. Hearing impairment associated with depression in US adults, National Health and nutrition examination survey 2005-2010. JAMA Otolaryngol Head Neck Surg. 2014;140(4):293-302.

35. Chia EM, Wang JJ, Rochtchina E, Cumming RR, Newall P, Mitchell P. Hearing impairment and health-related quality of life: the Blue Mountains hearing study. Ear Hear. 2007;28(2):187-95.

36. Barnett S, McKee M, Smith SR, Pearson TA. Deaf sign language users, health inequities, and public health: opportunity for social justice. Prev Chronic Dis. 2011;8(2):A45

37. Country Health Profile 2017. OECD/European Observatory on Health Systems and Policies. Brussels: State of Health in the EU, OECD Publishing, Paris/European Observatory on Health Systems and Policies. 2017.

38. McKee MM, McKee K, Winters P, Sutter E, Pearson T. Higher educational attainment but not higher income is protective for cardiovascular risk in deaf American sign language (ASL) users. Disabil Health J. 2014;7(1):49-55.

39. Health Interview Survey. 2014. http://www.statistics.gr/en/statistics/-/ publication/SHE22/- Accessed 7 Mar 2018.

40. Horner-Johnson W, Krahn GL, Suzuki R, Peterson JJ, Roid G. Hall T; RRTC expert panel on health measurement. Differential performance of SF-36 items in healthy adults with and without functional limitations. Arch Phys Med Rehabil. 2010;91(4):570-5.

\section{Ready to submit your research? Choose BMC and benefit from:}

- fast, convenient online submission

- thorough peer review by experienced researchers in your field

- rapid publication on acceptance

- support for research data, including large and complex data types

- gold Open Access which fosters wider collaboration and increased citations

- maximum visibility for your research: over $100 \mathrm{M}$ website views per year

At BMC, research is always in progress.

Learn more biomedcentral.com/submissions 